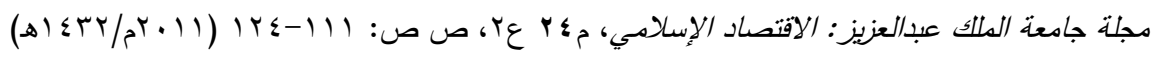
DOI: 10.4197 / Islec. 24-2.3

\title{
الندرة والمصلحة الخاصة والتعظيم من الزاوية الإسلامية مناقنشة منهجية لمحاضرة الأستاذ زبير حسن
}

\author{
عبدالرزاق سعيد بلعباس

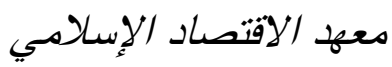 \\ جامعة الملك عبد العزبز، جدة - المدلكة العربية السعودية \\ abelabes@kau.edu.sa
}

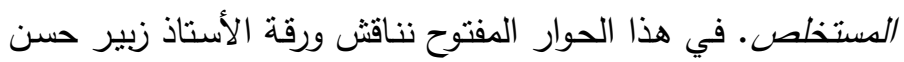

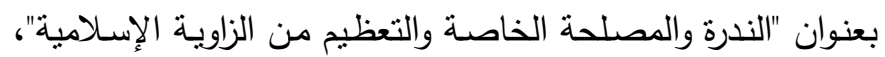

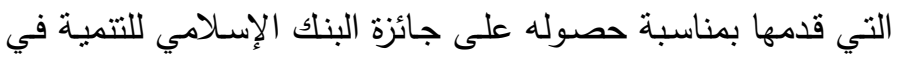

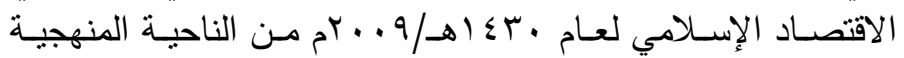

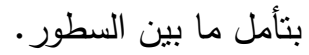

الكلمات الدفتاحية: المنهجية، الاقتصاد الإسلامي، النموذج النيوكلاسيكي،

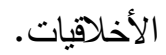

\section{مقدمة}

بعد أن شـهدت محاضـرة الأسـتاذ زبير حسـن التي ألقيت في 7 جمـادى الآخـرة

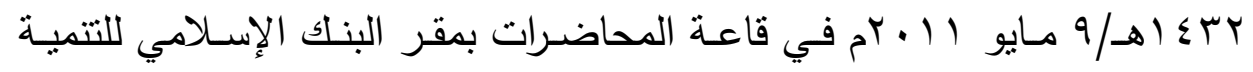

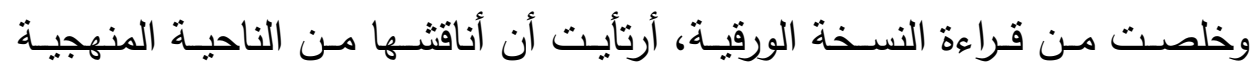

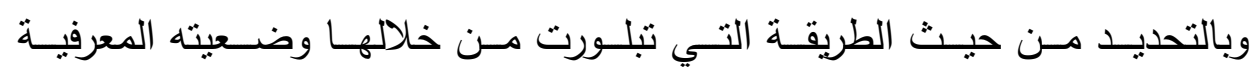


وخلفياتها وأسسها وأبعادها. (epistemological posture)

التسلسل الإدراكي

يرتكز طرح المحاضر على التسلسل الإدراكي (cognitive process) التالي:

لكي بضدن الاقتصاد الإسلامي وجوده ويُكتب له البقاء لا بد أن يصبح علمًا.

ولا يمكن لـه أن يحقق ذلك إذا استبتعد الأسـاس الذي بقوم عليـه علم الاقتصـاد باتجاهه السائد اليوم (صץ). ويتمنك هذا الأسـاس في ثثلثة مفاهيم محورية وهي الندرة والمصلحة الثخصية وتعظيم الربح (ص ا).

والمطلوب في تقدير المحاضر أن تستخدم هذه المفاهيم الأساسية بعد إعادة النظر في مضمونها وفحواها بحيث تتوافق مع معابير الثريعة الإسلامية (ص (Y).

مستوى التحليل

لم يفصح المحاضر عن وضعيته المعرفية من حيث مستوى التحليل: هل يندرج بحثه في التحليل الاقتصادي الجزئي أو البيني أو الكلي ؟ ومن خلال المفاهيم الرئيسـة التي تعالجها الورقة يتبين أن عرضسه يندرج في الاقتصـاد الجزئي. لكن أين تكمن أهمية تحديد هذا المستوى التحليلي ؟ أسس الاقتصاد بين المستويين التحليلين الجزئي والكلي

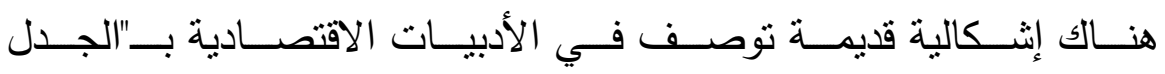
النيوكلاسيكيي- الكينزي" (Neoclassical-Keynesian Controversy) يتمحور حول طريقة التطرق إلى الظاهرة الاقتصادية: هل يتم ذلك بداية على المستوى الجزئي أم الكلي؟ وخلفية ذلك: هل يشكل الاقتصاد الجزئي أساسًا معرفيًا للاقتصاد الكلي أم العكس؟ 
يبدو أن المحاضـر، بحكم تكوينه الأصلـي (')، يميل إلى الـرأي الذي يـرى أن الاقتصاد الجزئي هو أساس الاقتصاد الكلي لأسباب فنية لأن النماذج الجزئية تعمل بشكل أفضل كلما انخفض عدد المتغيرات بينما يفترض الاقتصاد الكلي عددًا كبيرًا من المتغيرات والآليات رجعية المفعول (retroactions).

\section{موقع الاقتصاد الإسلامي من علم الاقتصاد بصيغته السائدة}

من مستلزمات هذا الطرح المعادلة المعرفية التالية التي تحدد موقع الاقتصـاد الإسلامي من علم الاقتصاد بصيغته السائدة: الاقتصاد الإسلامي ح علم الاقتصاد + الأخلاقيات الإسلامية

والاقتصـاد في تقدير المحاضر هو علم وضـي على غرار العلوم الدقيقة، وعلى هذا الأساس لا يوجد علم إسـامي. والمقصود بعلم الاقتصاد هنا في المقام الأول هـو الاقتصـاد الجزئي في صـيغته النيوكلاسـيكية القائمسة على مسـلمات

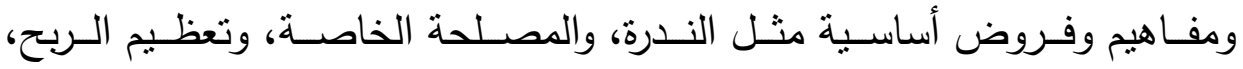
المطروحة بقوة في هذه الورقة. وهي تمثنل كما ذكر المحاضر (ص () مستشهدًا ببول سامويلسـون (Paul Samuelson) الأسـاس الذي أقيم عليهه علم الاقتصـاد

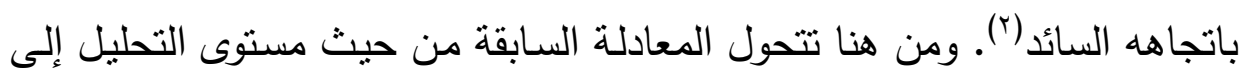

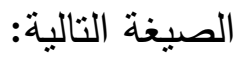
الاقتصاد الإسلامي ح النموذج الاقتصادي النيوكلاسيكي + الأخلاقيات الإسلامية (1) حصل المحاضر على دكتوراه في الاقتصاد عبر أطروحة عنوانها "نظرية الربح" من جامعة

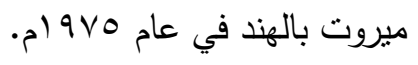

Paul Samuelson (1947) Foundations of Economic Analysis, Harvard University Press.( $($ ) تجدر الملاحظة بأن المحاضر لم يشر إلى هذا المرجع في مراجعه. 


\section{تعريف المحاضر للاقتصاد الإسلامي}

تظهر هذه العلاقة بشكل واضح عبر تعريف المحاضر للاقتصـاد الإسـامي: "هو الموضـوع الذي يدرس السـوك الإنسـاني فيمـا يتعلق بتعدد الحاجـات ونـدرة الموارد عبر الاستخدامات البديلة من أجل تعظيم الفلاح الذي يمثل الرفاه في الدنيا والآخرة"("). وهو تعريف برتكز على تعربف ليونيل روبينز (Lionel Robbins) لعلم الاقتصاد (๕)

ويتضـح من خـلال هذا التعريف أن المحاضر يعتبر أن سلوكيات الاقتصـاد النيوكلاسيكي تتطبق على الاقتصاد الإسـامي ما دامت لا تتعارض مع المعايير الإسلامية للسلوك (Hassan, 1992, p. 244)، وبالتالي فإن أوجه الاختلاف قد تطرأ على مستوى المدخلات أو "الاستخدامات البديلـة" (alternative use) والمخرجات أو الغايات، وبسميها "تعظيم الفلاح" (maximize falah). وتقترض هذه المقاربة أن الأدوات الضرورية لشرح ودراسـة الظواهر الاقتصادية

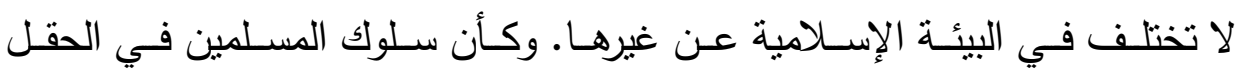
الاقتصادي في تصوره هو شكل من أشكال الرأسمالية. وحتى لو سلمنا بهذا التعميم

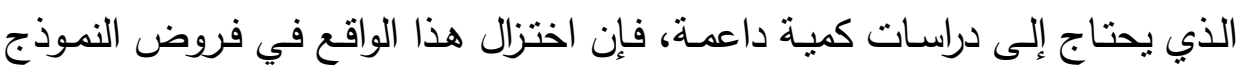
النيوكلاسيكي تبسيط مخل لأن الظاهرة الاقتصادية عملية معقدة تتداخل فيها عوامل (Rist, كثيرة تختلف من بيئة إلى أخرى. يقول الاقتصادي السويسري جيلبر ريست لائهن

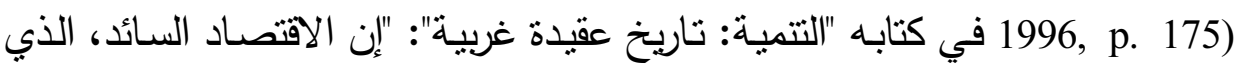

"Islamic economics is the subject that studies human behavior in relation to ( $\Gamma)$ multiplicity of wants and scarcity of resources with alternative uses so as to maximize falah that is the well-being in the present world and the hereafter" (p. 21).

"Economics is a science which studies human behavior as a relationship between ( $\varepsilon$ ) ends and scarce means which have alternative uses", Lionel Robbins (1945) An Essay on the Nature \& Significance of Economic Science, London: Macmillan, second edition revised and extended, p. 16. 
يُـرَّس اليـوم في الجامعـات، تبلور مسن خـلال ظـواهر تمـت ملاحظتهـا في الدول المنقدمة، التي تمنل بالتحديد حالة خاصة، وهي بالتالي غير قابلة للتطبيق على الحالة

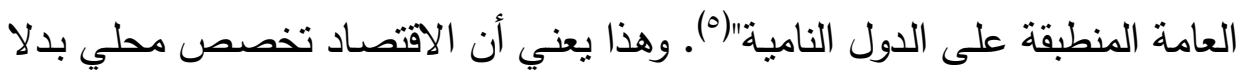
من أن يكون علمًا كونيًا، والأهم من ذللك هو معرفة الواقع الاقتصـادي وفهمهـ بقدر

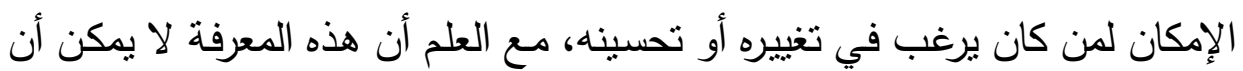
تكون إلا تقربيية مؤقتة تاريخيًا ومحددة الموقع جغرافيًا (Guerrien, 2004, p. 109).

\section{علاقة الاقتصاد الإسلامي بالاقتصاد النيوكلاسيكي}

لا يشك عاقل بأن الاقتصاد الإسـامي يلتقي مـع المدرسـة النيوكلاسيكية ليس الإسل في كل مفاهيمها ولكن في بعضها الذي يُعبِّر إلى حد ما عن السلوك الاقتصـادي مع العلم بأن هدف النيوكلاسيكيين الأساسي يكمن أكثر في تبرير كفاعة المنافسة

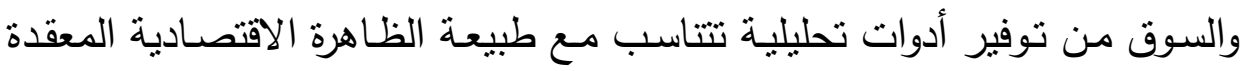
.(Guerrien, 2007)

ومن مستلزمات ذلك أن يبرز الاقتصـاد الإسـلاهي بكل وضـوح موقع هذه

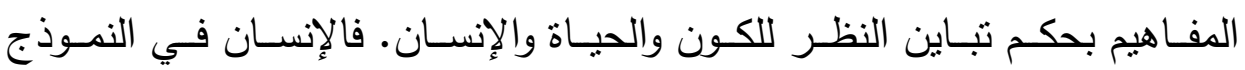
النيوكلاسيكي يُختزل في الفرد، والفرد في الفاعل (agent)، والفاعل في المنتج (أون الشركة) و /أو المستهلك الذي تُخوَّل لله درجة رضـا (satisfaction degree) ودالة منفعة (utility function). أما الاقتصاد الإسلامي، فيعتبر أن الإنسان جسم وعقل وروح خلق لعبادة الله عز وجل، وهذا يعني أن وظيفة المال لا تتحصر في إثباع

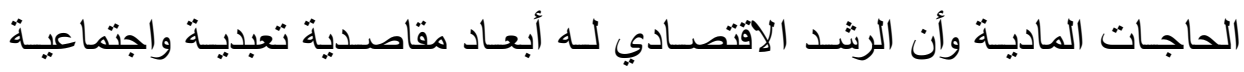
ونفسية لا تتفك عنه؛ مما يستدعي إدراج الاقتصاد في فضـائه المعرفي الأصلي لعيلي

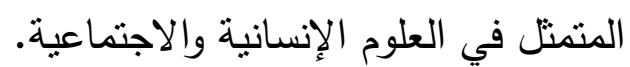

(0) نقل جيلبر ريست هذه الفكرة عن المرجع الثالي الذي لم أتنكن من الحصول عليه في الفترة المتاحة لي لكتابة هذه الورقة:

Dudley Seers (1963), The limitations of the Special Case, Bulletin of the Oxford University Institute of Economics \& Statistics, 25: 77-98. 


\section{فخ حيادية النمذجة الكمية}

بالرغم من تمكن المحاضر نسبيًا من أدوات التحليل الكمي، يبدو أنه وقع في

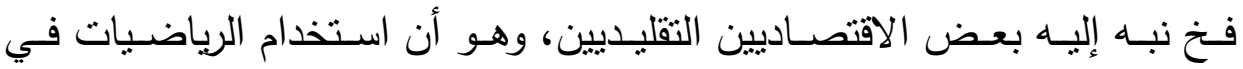

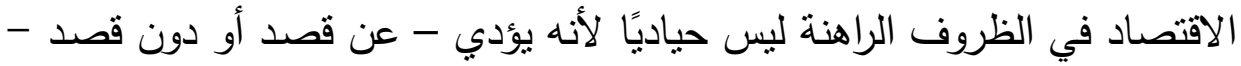
إلى تبني النموذج النيوكلاسيكي لنالثة عوامل أساسية وهي:

أولا: إن الفكر النيوكلاسيكي يحصـل منذ عقود على تموبـل كبير مـن قبل أنصار الإيديولوجية النيولييرالية (Légé, 2007).

ثانيـا: إن المدرسـة النيوكلاسيكية، بغض النظر عن موافقتها أو مخالفتها، قد

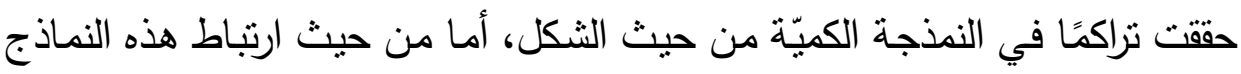
بالواقع فهذه قضية يصعب الدفاع عنها اليوم لأن استخدام الرياضيات في الاقتصاد قد

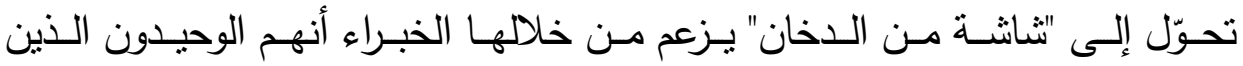
باستطاعتهم أن بعرفوا ما هو حاصل وما يجب حصوله وتجنبه (Guerrien, 2010). ثالثا: صعوبة نشر الأوراق في المجلات العلمية الذائعة الصيت إذا تعارض محتوى تللك الأوراق مع مسلمات المقاربة النيوكلاسيكية (d'Iribarne, 2009). عقدة التميّز يزعم الاقتصساديون النيوكلاسيكيون أن علم الاقتصاد يتميّز عن سائر العلوم

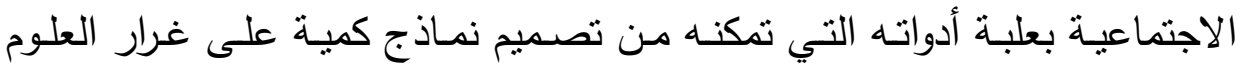

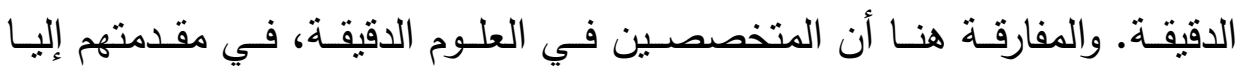
بريغوجين (Prigogine, 1996) (־) يدعون إلى تجديد العلوم من القناعات القديمة ومراجعـة الحتميـة (determinism) التي كانـت عند نيـونت (Newton) وأينشـتاين نظرًا لإستحالة التبؤ في الأنظمة الطبيعية بصفة مطلقة. ومعنى ذلك (Einstein)

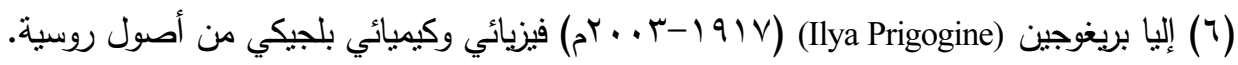

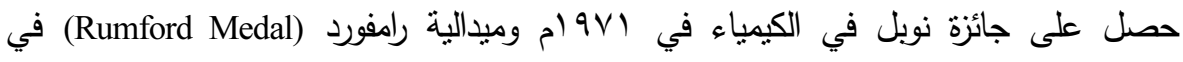
19VT ام التي تمنحها الجمعية الملكية البريطانية (Royal Society). 
استبدال المعادلات الحتميبة (deterministic equations) السـائدة في الفيزبـاء بحسابات إحصائية (statistical calculations) تعطي احتمالات (probabilities)، أي إن القوانين الأساسية لم تعد ثُشِّل يقينيات (certainties) وإنما فقط إمكانيات •(possibilities)

في ظل هذه التطورات الجديدة بات جليًا أن الاقتصادي لم يعد يتميز عن غيره إلا من حيث كونه يعرض خطابًا فنيًا قائمًا على مصطلحات بـات مثل الاستهلاك والإنتاج

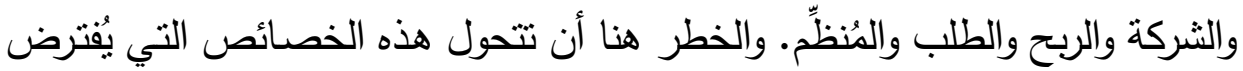

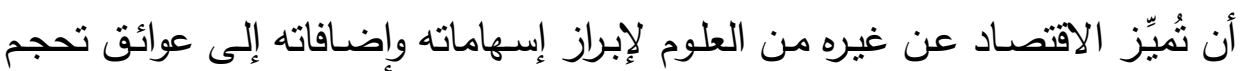
التفكير السليم. وفي هذه الحالة لا يمكن لعلم الاقتصاد أن بتقدم لأن التفكير السليم هو الإنها المحرك الأساسي والضروري لتقدم أي علم (Beaud, 1998). يبدو أن المتخصص في الاقتصاد الإسـامي ليس بمنأى عن عقلية من هذا

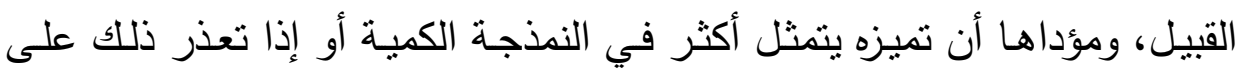
الأقل في مسلماتها ومفاهيمها وقوانينها الأساسية السائدة. وإذا كان بعضنا بعيب

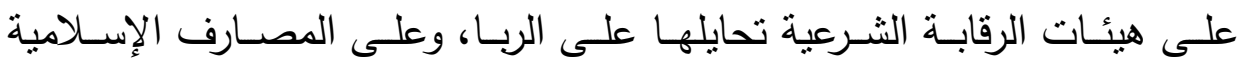

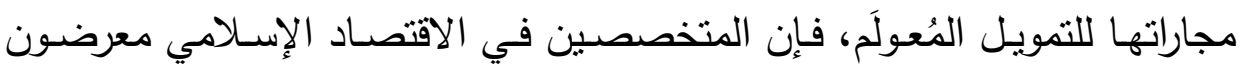
لهذا التحايل اللفظي والترقيع المعرفي الذي لا يُجْدي شيئًا بـل بُعقّد الأمور أكثر لئر ويُكرِّ الضبابية المنهجية والتبعية المعرفية تحت غطاء إسلامي.

\section{المنهجية الفردية}

إن المسألة الجوهرية لا تكمن في الفرضيات ككائنات معرفية فرعية بقدر ما أنها تُمُنّل جزءًا من منظومة. وهذا يعني أن هذه الفرضيات تستمد ثقلها المعرفي من انتمائها إلى منظومة قائمة على بنية خاصـة لها خلفياتها الفكرية ومنطالاقاتها الفلسفية التبريريـة. بعبـارة أخرى إن المنظومـة هـي التـي أبـرزت هذه الفرضـيات 
ضمن عملية انتقائية(V) نبلورت على مدار العقود الماضية وليس العكس. لذلك فإن حجر الزاوية في هذه المسألة هو تحديد موقع هذه الفرضيات في منظومة الاقتصاد

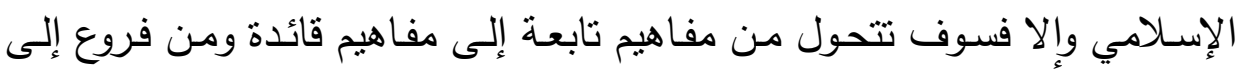
أصول تُهيكل التفكير وتحجم الإبداع.

إن تبني هذه الفروض مجتمعة بؤدي لا محالة إلى تبني المنظومة المعرفية

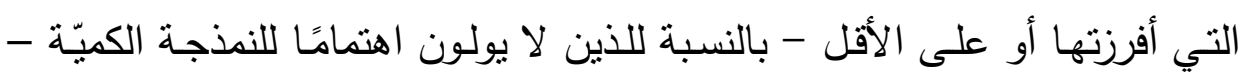

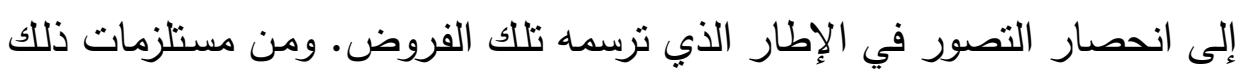
تبني المنهج الفردي (methodological individualism) الذي يتم بموجبه تفسير

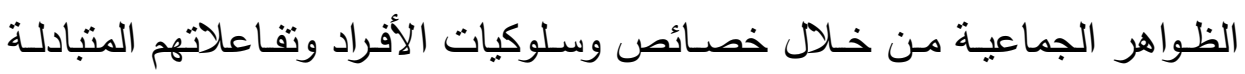

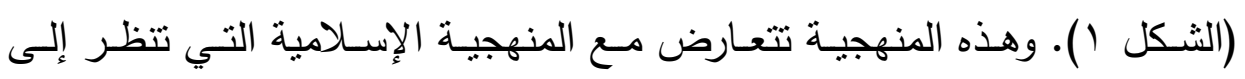

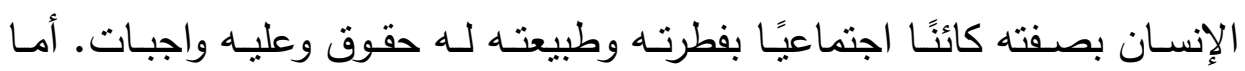
النموذج النيوكلاسيكي، فينفي الإنسان وعلاقته الاجتماعية وبيرز سيادة الفرد على المجتمع الذي لله حقوق وليس عليه واجبات ولا فرائض ويُكرَّس حتمبـة الخضوع للنظام الاقتصادي السائد (Caillé, 2005).

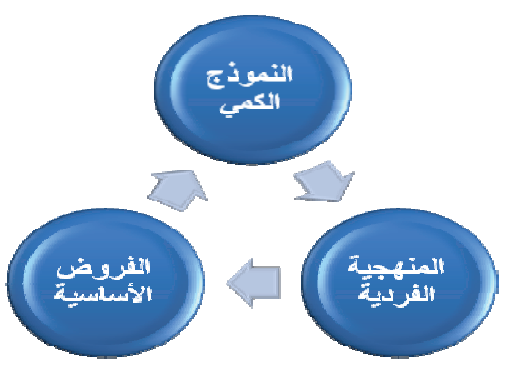

شكل (1) المنظومة المعرفية للنموذج النيوكلاسيكي.

(V) من ركائز هذه العملية الانتقائية استبعاد كل ما هو غير قابل للقياس. 


\section{تذريس الاقتصاد والاقتصاد الإسلامي}

إن المتأمـل للجدل القـائم في بعض الـدول الأوروبيـة، لاسبـيما في فرنسـا، بخصوص كيفية تدريس الاقتصاد في الجامعات على خلفية انحصارها في النموذج النيوكلاسيكي (Clerc, 2006) ليشعر بتقصير نسبي من قبل الباحثين في الاقتصاد

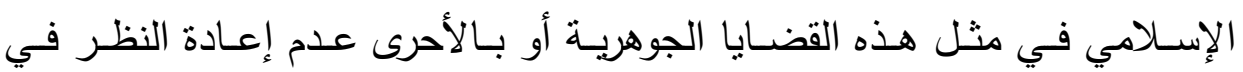
اجتهادات ظرفية حُدِّد مسارها في سياق معين.

إن الطريقة التي يتم من خلالها تدريس الاقتصاد الإسـامي في الفترة الراهنة

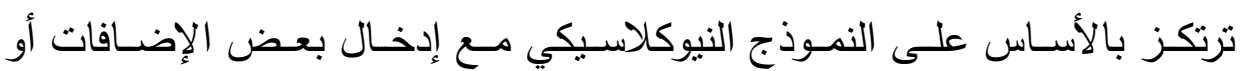
الأخلاقيات الإسلامية (Hanif, 2010) كلما تسنى ذلك من حيث المسألة المعالجة ومن حيث توفر النص وشروحه، لأن الاطلاع على النصوص وشروحها من قبل الراسخين في العلم(^) لا يزال في غير متتاول عدد كبير من الناطقين بغير العربية. ولعل المحاضر قد استقى تصوره للاقتصـاد الإسـلامي من هذه المقاربـة أو عزز قناعته الفكريـة مـن خلالها عند تدريسـه في الجامعـة الإسـامية العالميـة بماليزبـا واحتكاكه بالمهتمين بالاقتصاد الإسلامي في هذه المؤسسة العريقة طيلة م 1 سنة. إن أحسن طريقة لتدريس الاقتصاد الإسلامي، في ظل عدم التوصل إلى صباغة رصينة للنظرية الاقتصادية الإسـامية، هي الدراسة المقارنة التي تأخذ بعين الاعتبار خـارج التخصص المدارس المختلفة (النيوكلاسيكية، الكينزبـة، الاقتصـاد المؤسسي،

(^) استشهد المحاضر في عرضه على البوربنت في مستهل حديثه عن المصلحة الثخصية

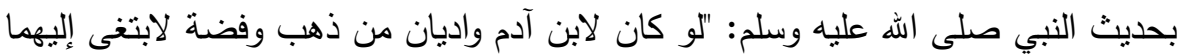

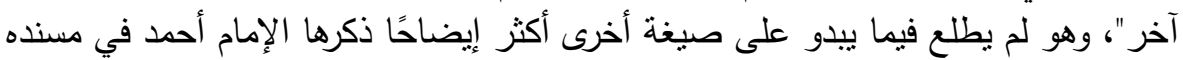

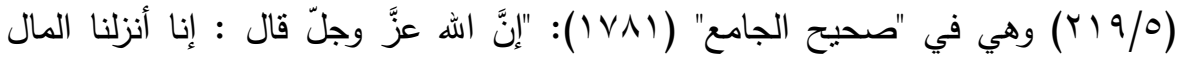

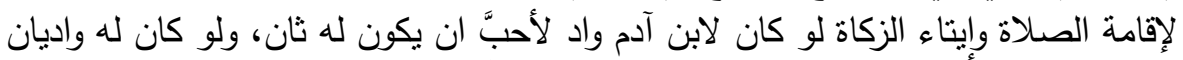

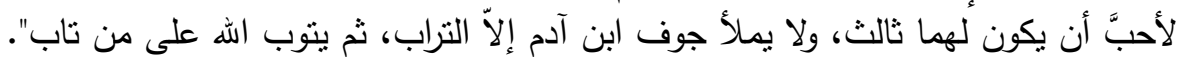

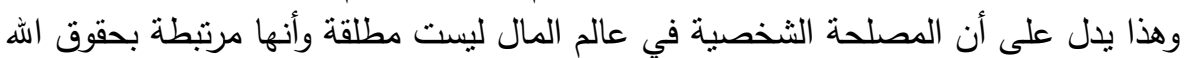

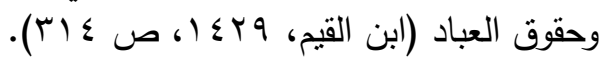


إلخخ)، ولا تغفل داخل التخصص تعدد المقاربـات في الاقتصـاد الإسـلامي (الفقهيـة، المعرفية، التحليّيّة، الكميّة، التاريخية، إلخ) حتى يتسنى توسيع آفاق الطلاب وتتمية قدراتهم النقديـة وتعويـدهم على تتـوع المقاربـات والمنــاظر وكيفيـة الاسـتفادة منهـا. فالاستتاد إلى الاقتصاد المؤسسي، على سبيل المثال، يكون الهدف منه جلب نظر الطلاب بأن لكل بلد بنية مؤسسية خاصة بـه، وعليه لا يمكن قبول فكرة الحل الفريد التي تغض النظر عن الخصائص المؤسسية. أما دراسة التركيبات المؤسسية، فتجلب النظر إلى أن العلاقات بين الفاعلين هي تركييات معقدة قائمة على المنافسة والصراع والتعاون والتعاضد، ومن ثم فمن الضـروري تحديد هذه العلاقات وتوجيهها لتحقيق الأهداف المنوطة بها.

\section{المعضلة المنهجية مرة أخرى}

يستحيل على أي علم أن بتقدم بـدون منهجيـة رصـينة يتحقق مـن خلالهـا

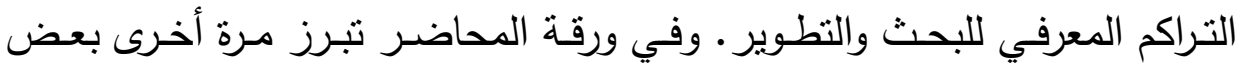
الاضطرابات المنهجيـة التي تواجـه الاقتصـاد الإسـلامي. ومعالجـة هذه المعضـلة عملية ليست بالهيِّة تستدعي تضافر جهود مختلف الأطراف ذات العلاقة بتحديد ماهيـة المنهجيـة ومسـتوياتها المختلفـة وقضـاياها الأساسية، وإدراجها في برنـامج بحث شـامل ومتكامل، والعمل على تكوين مجموعات بحثيـة متفاعلة ومنسـمة بغرض ربط الجزء بالكل وتقوبـة مخرجات المنتج المعرفي. وفي ظل غياب مستتد منهجي رصين، فإن مخرجات أبحاث الاقتصاد الإسـلامي سيغلب عليها الكم أكثر

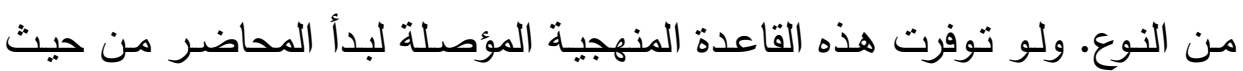
انتهى الآخرون ولاستفاد الاقتصاد الإسلامي منه بطريقة أفضل.

وبقدر ما أختلف مـع المحاضر بصدد مقاربته المعرفية، فإنني أتفق معه في كون النظام الاقتصـادي الإسـلامي في نهايـة المطاف ليس مـا يُكتب مـن خـلال الأمنيات والمثاليات، ولكن ما يُطبق على أرض الواقع (ص 0). بعبارة أخرى إن إن 
إخبار المره عن طبيعة النظام الاقتصـادي الذي يؤمن بـه شيء مهم، لكن الأهم من ذلك هو ما يفعله هذا النظام به وبواقعه الفعلي.

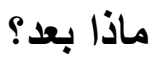

إن غياب تطبيق الاقتصاد الإسـامي على المستوى الكلي يحرم الباحثين من دراسة واقعهم "كما هو" من خلال بلورة فرضيات وتمحيصها على ضوء المعطيات الكمية المتوفرة واستخراج نتائج تُفنّد أو تُؤكد هذه الفرضيات حتى يتم تدريجيًا بناء نظريـة متماسكة تُجسِّد الواقع ليتسـنى للقائمين على السياسـات إصـلاحه وتطوبره بالتنسيق مع الأطراف ذات العلاقة.

في ظل هذه الاستحالة المعرفيـة النسبية، يتعين على الباحثين دراسـة تطبيقات الاقتصاد الإسلامي في المجالات المتاحة على المستوين الجزئي(9) (microeconomic) والبيني('(mesoeconomic) (بناء على الفحص الفعلي والتحليل النظري التطبيقي الذي يأخذ بعين الاعتبار توفر الثروط وانتفاء الموانع.

وهنا لا بد من التقريق بين استخدام الأساليب الكميّة وتبني نماذج الاقتصاد القياسي السائدة التي لا تعكس الواقع وتتحصر في العلاقات الخطية التي تستبعد كل مـا هو غير قابـل للقياس وتتفي بالتالي الظواهر المعقدة والخصوصيات العقائدية والثقافية والاجتماعية وما يتمخض عنها من تعددية في النظرة (vision) والمعقولية(') ('rationality)

(9) منل المصارف وشركات الاستثمار والتأمين الإسلامية. (1) (1) مثل قطاعات الزكاة والوقف والتمويل الإسلامي.

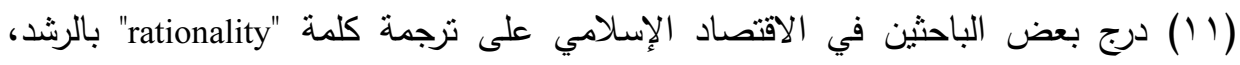

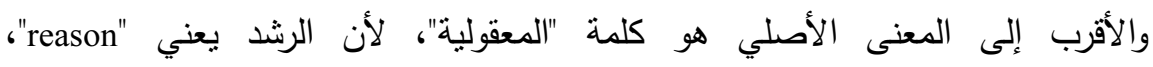

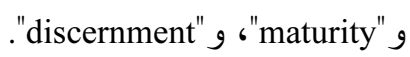


في مقابل ذلك، إن التصورات الجديدة الناجمة عن تطور العلوم التي لا تهمل ظـواهر عـدم الاسـتقرار (instability) والتقلبـات (fluctuations) والفوضـى (chaos) والاضطراب (disorder) باتت ضرورية للوصول إلى فهم أفضل للواقع المعقد والتعامل معـه بجديـة لتعظيم المصـالح وتقليـل المفاسـد بقدر الإمكـان. ومـن هنـا تأتي أهميـة المقاربـة متعـددة التخصصــات (systemic approach) التـي تــولي اهتمائًا أكبـر

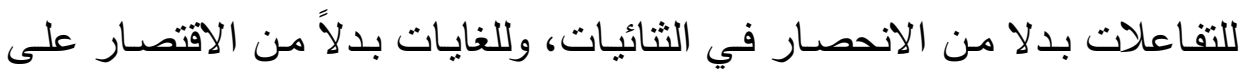

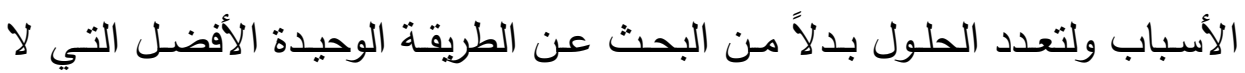
تراعي ظـروف الزمـان والمكـان والحسال كمـا هـو حاصـل في النمـوذج الخطي .(Donnadieu et al., 2003)

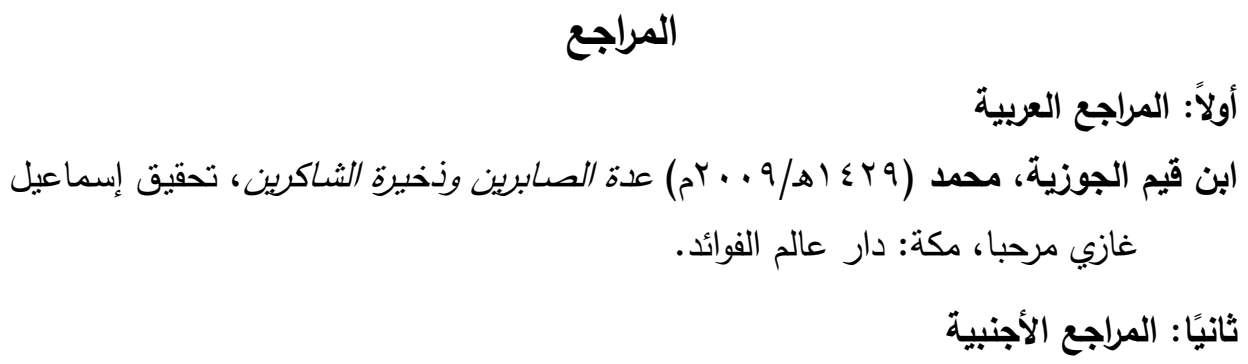

Aslam, Hanif Mohammed (2010) Teaching Economics at the International Islamic University Malaysia, Wednesday Seminar No. 8, Jeddah: Islamic Economics Institute, December, 29.

Beaud, Michel (1998) Y a-t-il encore place pour la pensée dans l'univers des économistes universitaires?, in François Michon, L'économie, une science pour l'homme et la société : mélanges en l'honneur d'Henri Bartoli, Publications de la Sorbonne, Paris, :21-36.

Caillé, Alain (2005) Dé-penser l'économique. Contre le fatalisme, La Découverte / M.A.U.S.S., Paris.

Clerc, Denis (2006) L'enseignement de l'économie en France, Nouvelles Fondations, 2006/2, :76-82, http://www.cairn.info/revue-nouvelles-fondations-2006-2-page76.htm

Donnadieu, Gérard, Durand Daniel, Neel Danièle, Nunez Emmanuel et Saint-Paul Lionel (2003) L'Approche systémique: de quoi s'agit-il? Synthèse des Travaux du Groupe AFSCET 'Diffusion de la pensée systémique', septembre, http://www.afscet.asso.fr/SystemicApproach.pdf

Guerrien, Bernard (2004) Y-a-t-il une science économique?, L'économie politique, 2004/2, No. 22, :97-109, www.cairn.info/load_pdf.php?ID_ARTICLE=LECO -022_0097 
Guerrien, Bernard (2007) Que penser des outils de la théorie néoclassique?, L'économie politique, avril, No.73.

Guerrien, Bernard (2010) Le rôle des mathématiques en économie, Contretemps, http://www.contretemps.eu/interventions/sur-role-mathematiques-en-economie

Hassan, Zubair (1992) Profit maximization: secular versus Islamic, Sayyed Tahir, Aidit Ghazaly and Syed Omar Syed Agil, Readings in Microeconomics: An Islamic Perspective, Longman Malaysia, pp: 239-255.

Hassan, Zubair (2011) Curriculum Vitae, Kuala Lumpur: INCEIF, The Global University of Islamic Finance, http://www.inceif.org/why-inceif/facultystrength/148

Hassan, Zubair (2011) Scarcity, Self-interest and Maximization from Islamic Angle, IDB Prize Winners' Lectures Program, Jeddah: Islamic Development Bank.

Iribarne, Philippe d' (2009) Comment interroger les postulats fondateurs de l'économie ?, Université d'été de l'Institut des Hautes Etudes pour la Science et la Technologie, 7 septembre, http://www.ihest.fr/activites/universite-europeenned-ete/comment-interroger-les-postulats

Légé, Philippe (2007) Théorie néoclassique : les débuts d'une domination, Alternatives Economiques, Hors Série No. 73, L’histoire de la pensée économique, avril, pp: 24-25.

Rist, Gilbert (1996) Le développement, histoire d'une croyance occidentale, Editions Sciences Po, Paris.

Robbins, Lionel (1945) An Essay on the Nature \& Significance of Economic Science, London: Macmillan, second edition revised and extended.

Samuelson, Paul (1947) Foundations of Economic Analysis, Harvard University Press. 


\title{
Scarcity, Self-interest and Maximization from Islamic Angle A Methodological Discussion of Zubair Hasan's Paper
}

\author{
Abderrazak Said Belabes \\ Islamic Economics Institute \\ King Abdulaziz University, Jeddah, Saudi Arabia \\ abelabes@kau.edu.sa
}

\begin{abstract}
In this open dialogue, we discuss the paper of professor Zubair Hasan entitled "Scarcity, Self-interest and Maximization from Islamic Angle", which he presented at the Islamic Development Bank on the occasion of his winning of the IDB Prize in Islamic economics for the year 2009. The discussion is carried out through a methodological reading between the lines.
\end{abstract}

Keywords: Methodology, Islamic economics, neoclassical model, ethics. 